\title{
Last But Not Least: Cortical Interneurons from Caudal Ganglionic Eminence
}

\author{
Gaia Colasante ${ }^{1,2}$ and Alessandro Sessa ${ }^{1,2}$ \\ ${ }^{1}$ Molecular Medicine (section: Neuroscience) PhD Program, "Vita e Salute" San Raffaele University, 20132 Milan, Italy, and ${ }^{2}$ Stem Cell and Neurogenesis \\ Unit, Division of Neuroscience, San Raffaele Scientific Institute, 20132 Milan, Italy \\ Review of Miyoshi et al.
}

Mammalian cerebral cortex is composed of excitatory glutamatergic principal neurons ( $\sim 80 \%$ of the total) and inhibitory GABAergic interneurons ( 20\%). Although GABAergic interneurons are less abundant, they have a crucial role in regulating cortical excitability and ultimately, in ensuring the physiological functioning of the whole brain. In fact, alterations in the ratio between glutamatergic and GABAergic tones lead to neurological disorders ranging from epilepsy to autism and schizophrenia (Lewis et al., 2005).

During brain development glutamatergic and GABAergic neurons arise from different compartments: principal neurons originate in the ventricular zone of the developing cortex and migrate radially, whereas GABAergic neurons derive, at least in rodents, exclusively from the ganglionic eminences (GEs) of the ventral forebrain and reach the cortex through tangential migration (Marín and Rubenstein, 2001).

GABAergic interneurons of the cerebral cortex are a heterogeneous population and the differences between subtypes are functionally relevant. In the last several years, great effort has gone into defining classification criteria, an essential step toward understanding how inhibition shapes cortical function (Ascoli et al.,

Received April 1, 2010; revised April 21, 2010; accepted April 23, 2010. Correspondence should be addressed to either Gaia Colasante or Alessandro Sessa, San Raffaele Scientific Institute, Via Olgettina 58, 20132 Milan, Italy, E-mail: colasante.gaia@hsr.it or sessa.alessandro@hsr.it. D0I:10.1523/JNEUROSCI.1677-10.2010

Copyright $\odot 2010$ the authors $\quad 0270-6474 / 10 / 307449-02 \$ 15.00 / 0$
2008). Up to now, cortical interneurons have been categorized based on molecular features, electrophysiological properties, dendritic arbor shape, and axonal targets. Defining developmental features, such as place of origin, date of birth, mode of migration, and final intracortical position of distinct classes of interneurons, might also be helpful for classification.

Several recent genetic fate mapping studies have been investigated the temporal and spatial origin of interneuron subclasses in the ventral telencephalon. In 2007, the Kessaris laboratory demonstrated that cortical interneurons expressing calbindin, parvalbumin, or somatostatin, as well as Martinotti cells (coexpressing calretinin and somatostatin) are generated exclusively from Lhx6-expressing precursors in the medial ganglionic eminence (MGE) (Fogarty et al., 2007). In contrast, all bipolar calretinin-expressing neurons, and most NPY-expressing interneurons, are generated outside the MGE, in the Gsh2+ germinal zones of the lateral/caudal ganglionic eminences (LGE/CGE). According to Fogarty et al. (2007), the MGE generates $\sim 70 \%$ of all the cortical interneurons, whereas the CGE generates the remaining 15\%. More recently, Gelman et al. (2009) identified the preoptic area (POA) as a novel source of GABAergic interneurons.

A recent Journal of Neuroscience paper by Miyoshi et al. (2010) fits very well in this context and provides new, important findings based on genetic fate mapping of CGE-derived cortical interneurons. The genetic strategy involved expressing Cre recombinase under a specific promoter (Mash1) that is activated during the early stages of interneuron specification in mice. Administering tamoxifen induced recombinase activity, which resulted in genetic GFP labeling of the Mash1-expressing cells and their progeny [Miyoshi et al. (2010), their Fig. $1 \mathrm{~A}$ ]; Cre recombinase activation at different times allowed the authors to label cohorts of cells born at different times.

Mash1 is normally expressed in all precursor cells in the ventral forebrain, but the Mash $1::$ Cre ER mouse line used in this work expresses the transgene only in CGE and LGE; no Cre-mediated recombination was detected in MGE [Miyoshi et al. (2010), their Fig. $1 E, F]$. The authors cleverly exploited this property of the transgenic line to fate map only the CGE-derived cortical interneurons. In fact, the authors claimed that they only mapped CGE-derived neurons, because the LGE does not contribute to cortical interneuron population. However, this issue is not entirely settled. Wichterle et al. (2001) showed that LGE cells migrate ventrally and anteriorly, generating GABAergic neurons in the striatum, nucleus accumbens, and olfactory bulbs, but LGE-derived interneurons did not invade the cortical plate in embryonic day 13.5 (E13.5) embryos. But Anderson et al. (2001), tracking the fate of interneurons derived from BrdUlabeled LGE neuroepithelium transplanted into the basal telencephalon of unlabeled brain slices, observed that although interneurons derived from E12.5 LGE did not 
migrate to the cortex, cells derived from E14.5-E16.5 transplants gave rise to interneurons reaching the cortical plate.

Miyoshi et al. (2010) accurately verified the origin of intracortical neurons labeled by tamoxifen injections starting at E12.5. As expected, from previous observations (Anderson et al., 2001; Wichterle et al., 2001), fate-mapped cells originated within the CGE, as deduced by their lack of Lhx6 expression (which labels MGE and LGE), while expressing CoupTFII (a marker of CGE) [Miyoshi et al. (2010), their Fig. 2]. However, the authors did not verify the origin of intracortical interneurons specified by tamoxifen administration at E14.5-E18.5. In this time window, contribution from the LGE compartment is possible (Anderson and al., 2001), so the authors should have more properly defined the fate-mapped interneurons as CGE/LGE derived. The authors demonstrated that CGE starts producing interneurons later than MGE (E12.5 vs E9.5) and the peak of production is delayed as well (E16.5 vs E13.5); also this finding did not exclude that this peak of interneuron production might include LGE-derived cells.

Moreover, they shed new light on the intracortical distribution of CGE/LGE interneurons. Their findings indicated that, unlike MGE-derived cortical interneurons, which after migrating tangentially distribute radially in the cerebral cortex in an "inside-out" fashion (Wonders and Anderson, 2006; Batista-Brito and Fishell, 2009), CGE/LGE-derived cortical neurons almost exclusively populate the most superficial layers regardless of their birthdate [Miyoshi et al. (2010), their Fig. 3C]. The authors suggest that future experiments, such as heterochronic transplantations, can eventually demonstrate whether the preferential disposition of late migrating interneurons (both from MGE and CGE/ LGE) in the upper part of the cortex results from their intrinsic migration properties or from a change into the intracortical cues.

In this regard, it could be interesting to investigate the evolutionary meaning of the coupling between the last-born glutamatergic neurons, constituting the upper layers, and the last-born interneurons, which Miyoshi et al. (2010) demonstrated to be mainly derived from CGE/LGE. It is well known that in the transition between sauropsids (birds and reptiles) and mammals, the cerebral cortex expanded from three to six layers (Molnár et al., 2006). Might the CGE/LGE interneuron population have evolved to increase the inhibitory pool in that enlarged cortex? To assess this issue, analyzing the migration pattern from the caudal part of the subpallium in animals with a three-layered cortex may be helpful, if feasible. In the future, it would be informative to investigate the timing, radial movements after tangential migration, and dispersion along the rostrocaudal axis of CGE/LGE interneurons using the powerful genetic tools established in this study.

Having established the time course of neurogenesis in CGE/LGE, Miyoshi et al. (2010) next produced a tremendous effort to systematically characterize CGE/LGEderived interneurons based on the Petilla Terminology guidelines (Ascoli et al., 2008). They defined different subclasses on the basis of biochemical markers [Miyoshi et al. (2010), their Fig. 5] establishing some hallmarks, for instance, low levels of Mash1 expression are present in migrating CGE/LGE interneurons [Miyoshi et al. (2010), their Fig. 2F], whereas either Reelin or VIP (vasoactive intestinal peptide) labels almost all mature CGE/LGEderived interneurons. Next, the authors divided all these interneurons into nine distinct subtypes, according to electrophysiological and morphological analysis [Miyoshi et al. (2010), their Fig. 6 and Table 4].

The work presented by Miyoshi et al. (2010) gives new and important insight into cortical GABAergic interneuron diversity, better defining the CGE/LGE-derived population. Through a genetic approach, they revealed the fate map of these cells and provided a detailed morphological, biochemical, and electrophysiological characterization. Moreover, their findings indicate that CGE/LGE-derived interneurons represent a surprisingly large part of all cortical interneurons ( $30 \%$ vs $\sim 15 \%$ estimated up to date). These data are valuable not only for a deeper knowledge of the complex landscape of cortical interneurons in physiological state but also to analyze more efficiently specific alterations in disorders or animal models of disease.

The connections of CGE/LGE-derived neurons remain to be investigated: What inputs do they receive? What neurons do they mainly target? Is their neuronal activity essential for particular networks? Likely, they participate in local circuits in the upper lay- ers, but it would be interesting to go deeper in this aspect, even if technically very demanding.

Finally, the GEs are also the source of GABAergic neurons that populate the ventral telencephalon. In this regard, another interesting issue for future work regards the analysis of the specific contribution of the CGE/LGE-derived neurons to basal ganglia structures in comparison with MGE- or POA-derived interneurons.

\section{References}

Anderson SA, Marín O, Horn C, Jennings K, Rubenstein JLR (2001) Distinct cortical migrations from the medial and lateral ganglionic eminences. Development 128:353-363.

Ascoli GA, Alonso-Nanclares L, Anderson SA, Barrionuevo G, Benavides-Piccione R, Burkhalter A, Buzsáki G, Cauli B, Defelipe J, Fairén A, Feldmeyer D, Fishell G, Fregnac Y, Freund TF, Gardner D, Gardner EP, Goldberg JH, Helmstaedter M, Hestrin S, Karube F, et al. (2008) Petilla terminology: nomenclature of features of GABAergic interneurons of the cerebral cortex. Nat Rev Neurosci 9:557-568.

Batista-Brito R, Fishell G (2009) The developmental integration of cortical interneurons into a functional network. Curr Top Dev Biol 87:81-118.

Fogarty M, Grist M, Gelman D, Marín O, Pachnis V, Kessaris N (2007) Spatial genetic patterning of the embryonic neuroepithelium generates GABAergic interneuron diversity in the adult cortex. J Neurosci 27:10935-10946.

Gelman DM, Martini FJ, Nóbrega-Pereira S, Pierani A, Kessaris N, Marín O (2009) The embryonic preoptic area is a novel source of cortical GABAergic interneurons. J Neurosci 29: 9380-9389.

Lewis DA, Hashimoto T, Volk DW (2005) Cortical inhibitory neurons and schizophrenia. Nat Rev Neurosci 6:312-324.

Marín O, Rubenstein JLR (2001) A long, remarkable journey: tangential migration in the telencephalon. Nat Rev Neurosci 2:780-790.

Miyoshi G, Hjerling-Leffler J, Karayannis T, Sousa VH, Butt SJB, Battiste J, Johnson JE, Machold RP, Fishell G (2010) Genetic fate mapping reveals that the caudal ganglionic eminence produce a large and diverse population of superficial cortical interneurons. J Neurosci 30:1582-1594.

Molnár Z, Métin C, Stoykova A, Tarabykin V, Price DJ, Francis F, Meyer G, Dehay C, Kennedy H (2006) Comparative aspect of cerebral cortical development. Eur J Neurosci 23:921-934.

Wichterle H, Turnbull DH, Nery S, Fishell G, Alvarez-Buylla A (2001) In utero fate mapping reveals distinct migratory pathways and fates of neurons born in the mammalian forebrain. Development 128:3759-3771.

Wonders CP, Anderson SA (2006) The origin and specification of cortical interneurons. Nat Rev Neurosci 7:687-696. 